\title{
Observation of Anisotropic Emission from Semiconductor Quantum Dots in Nanocomposites of Metal Nanoparticles
}

\author{
Tuncay Ozel, ${ }^{1}$ Sedat Nizamoglu, ${ }^{1}$ Mustafa A. Sefunc, ${ }^{1}$ Olga Samarskaya, ${ }^{1}$ Ilkem O. Ozel, ${ }^{1}$ Evren \\ Mutlugun, ${ }^{1}$ Vladimir Lesnyak, ${ }^{2}$ Nikolai Gaponik, ${ }^{2}$ Alexander Eychmuller, ${ }^{2}$ Sergey V. Gaponenko, ${ }^{3}$ and \\ Hilmi Volkan Demir ${ }^{1,4, *}$ \\ ${ }^{1}$ Department of Physics, Department of Electrical and Electronics Engineering, and UNAM - Institute of Materials Science and Nanotechnology, \\ Bilkent University, TR-06800, Ankara, Turkey, ${ }^{2}$ Physical Chemistry, TU Dresden, Bergstr. 66b, 01062 Dresden, Germany , ${ }^{3}$ Stepanov Institute of \\ Physics, National Academy of Sciences of Belarus ， 220072 Minsk, Belarus , ${ }^{4}$ Nanyang Technological University, School of Electrical \& \\ Electronic Engineering, Divi sion of Microelectronics, School of Mathematical and Physical Sciences, Luminous! Semiconductor Lighting and \\ Display Center of Excellence, Nanyang Avenue, Singapore 639798, Singapore \\ *Tel:[+90]( 312) 290 1021, e-mail: volkan@stanforalumni.org
}

Colloidal semiconducting quantum dots ( QDs), also known as nanocrystals, offer a number of advantages in light generation, e.g., for solid-state lighting, biol ogical labeling, and bio -imaging. ${ }^{3}$ These applications typically necessitate high quantum eff iciencies in film, preferably with polarized emission in certain applications. One of the major problems encountered when working with films of these QDs is the decrease in their fluorescence quantum efficiency in the solid for $\mathrm{m}$. Another technical challenge is to obtain anis otropic emission from these QDs. Recently plasmonics has enabled a wide range of important applications related to nanocrystals including metal enhanced light generation and plasmonic biosensing. ${ }^{1,2}$ In this work, to address the se difficulties encountered with QD solids, we investigate and demonstrate anisotropic enhanced emission from semiconductor quantum dots placed in multilayered plasmon resonator nanocomposite $\mathrm{s}$ using strong plasmon-exciton interactions . In these metal nanoparticle (MNP) resonator architectures, we show that highly isotropic nature of QD emission c an desirably be altered through modifying emission kinetics by plasmon coupling, while also enhancing the overall QD emission.

To date plasmo $\mathrm{n}$ coupling of such QDs has been widely studied using various methods including the self-assembly of a monolayer of QDs and a monolayer of M NPs. However, previous works studied only the plasmonic coupling of a QD film located either on top or bottom of the metal nanostructure layer, focusing on the resulting bilayer systems of MNPs and QDs . On the other hand, the emission polarization has been investigated and observed for only anti symmetric particle systems such as semiconductor nanorods. Different than the previous reports, here our work focuses on the demonstration of plasmon -coupled QD solids in a three -dimensional construction by repeating QD MNP unit cells to increase the quantum efficiency of the composite film and modify the isotropic emission nature of QDs in plasmonic cavities. In our bottom -up approach, the polarization properties are significantly modified , resulting in a $80 \%$ of emission in vertical polarization, and a quantum efficiency of $30 \%$ is achieved for the se plasmonic QD-composite films.
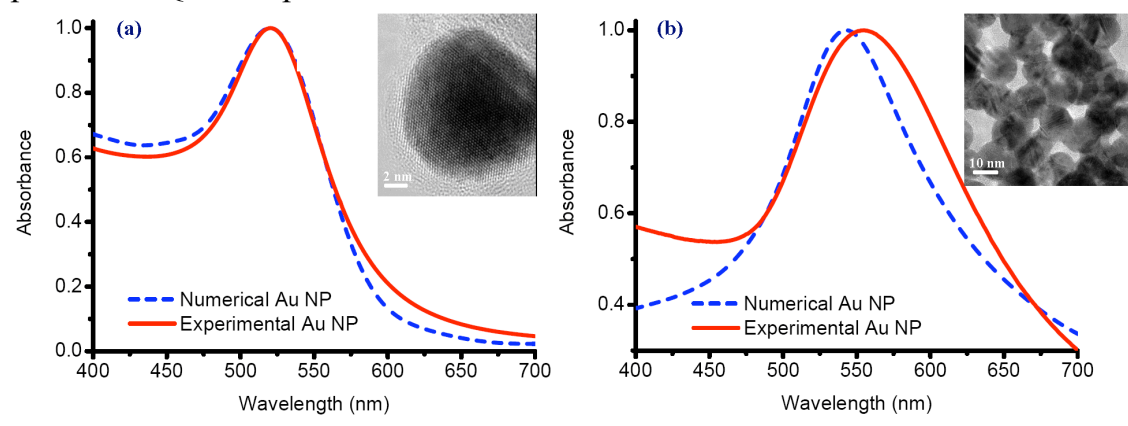

Figure 1. Simulation and experimental results for normalized absorbance spectra of (a) isolated

assembled particles in a dielectric medium. Insets are transmission electron microscopy images of a single Au nanoparticle in (a) and close-packed assembly in film in (b). The respective scale bars are $2 \mathrm{~nm}$ (a) and $10 \mathrm{~nm}$ (b). (c) Time-resolved fluorescence decay curves of CdTe QD composite film alone and in the presence of Au nanoparticles in the composite, all recorded in vertical (V...) excitation polarization using vertical ( ...V) or horizontal ( ...H) polarizer orientations of emission collection setup. The legend shows the first "V..." for vertical excitation polarization and the second "...V"/“...H" for the vertical/horizontal emission.

Plasmonic coupling of CdTe QD layer using Au nanoparticles in a bilayer system has been previously reported both for on-resonance ${ }^{2}$ and off-resonance ${ }^{3}$ spectral conditions. However, one of the most important conditions for strong plasmon-exciton interaction $\mathrm{s}$ is the spectral overlap between plasmon resonance wavelength of MNPs and emission wavelength of QDs. In our experiments, w e observe strong on-resonance plasmon coupling of CdTe QDs, which is also supported by o ur numerical calculation s. As depicted in Fig . 1(a)-(b) there is a significant red -shift in the localized plasmon resonance wavelength of MNPs when they are cast into a closely-packed film. In our design, w e 
intentionally use short gaps between MNP layers in each plasmon resonator since the generated local ized electric field is increased as the gap in the resonator is decreased. This is also supported by our simulation results, and with very recent theoretical and computational calculations in the literature. ${ }^{4-5}$ Electric field intensity decays rapidly in a single unit cell (Fig. 2(a)) but increases and elongat es through separation gap in the case of 2 - and 5-times repeating cavity-forming unit cells (Fig. 2(b)-(c)). In our proposition, CdTe QD layers are exposed to dramatically stronger plasmonic coupling when placed in these plasmonic resonators made by vertically layer-by-layer assembl ing of several QD and MNP layers in groups on top of each other.
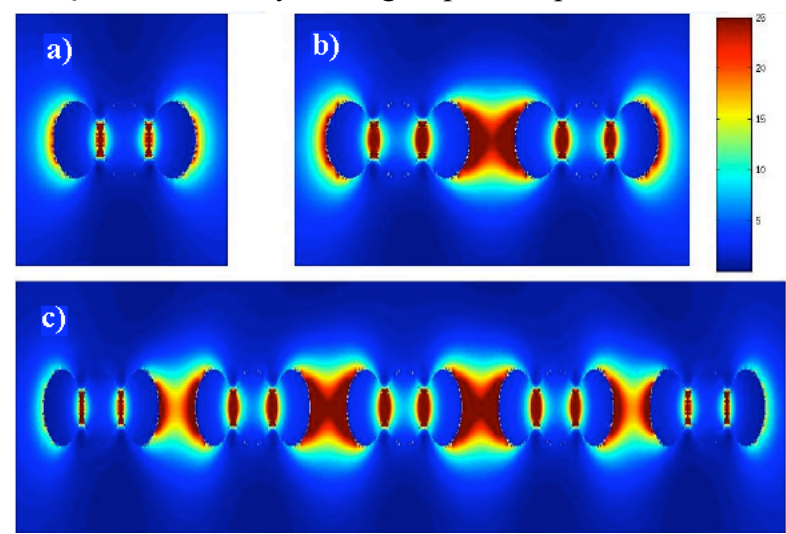
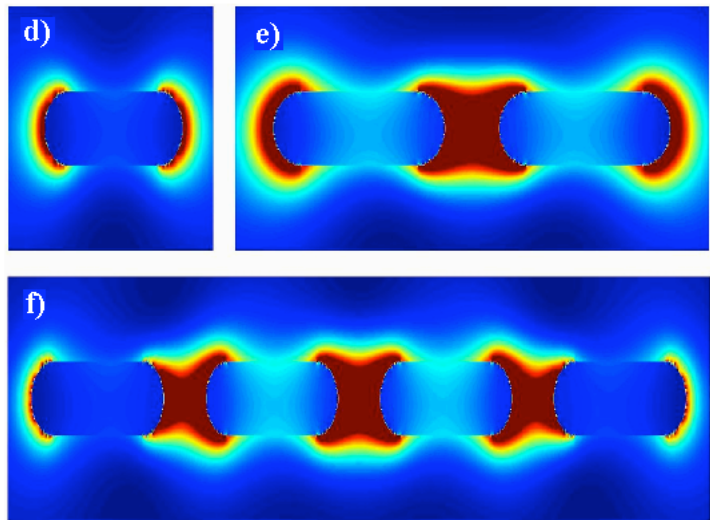

Figure 2. Electric field intensity map distribution of (a) 1 -, (b) 2 -, and (c) 5-times repeating hori zontally aligned three $\mathrm{Au}$ nanoparticles and (d) 1, (e) 2, and (f) 4 Au nanorods with half sphere ends. (Here note that the field maps shown in this figure are rotated by $90^{\circ}$ with respect to the orientation of the composite film samples, for which the horiz ontally side -by-side metal nanospheres depicted here correspond to vertically aligned nanoparticles in the composite film s.)

We use a polarizer -integrated time -correlated single photon counting system with precis e cut-off filters for anisotropy measurement s. Fig. 1(c) shows time -resolved fluorescence decay curves of CdTe QD composites, all recorded in the same vertical excitation polarization but in different orientations of collection polarization. The emission kinetics of these QD solids is highly modifie $d$ when they are embedded in the coupled plasmonic resonator structure. Using Picoquant FluoFit software we calculated the estimated steady-state an isotropy value of the CdTe QD films, which is increased from 0.02 to 0.26 in the presence of plasmon resonato $r$ structures as a result of artificial three-dimensional film construction. By integration of the decay curves over time, we measure $80 \%$ of the emission is in the vertical polarization and the total emission enhanced by a factor of 4.4 folds as a result of plasmon coupling in this composite film. In this plasmonic composite architecture, three monolayers of metal spheres are assembled one after the other as one group in vertical direction (with less than $1 \mathrm{~nm}$ spacing) before placing QDs in their cavity. Surface characterization via a tomic force microscopy for this three-monolayer Au nanoparticles film shows that they are placed on top of each other in vertical direction, with some having 2 particles and with some having 3 particles on top of each other. These MNPs then serve together as a group like metal nanorods. As depicted in Fig. 2 (a), 2 (b), and 2 (c), the group of triple Au nanoparticle simulation results match well with half sphere end Au nanorod simulations given correspondingly in Fig 2 (d), 2 (e), and 2(f). This shows that nanorod-like anti-symmetrical localized surface plasmons are generated from the groups of spherically symmetric Au NPs.

In conclusion, using our artificial three -dimensional plasmon resonator nanocomposite structure , the emission characteristics of CdTe quantum dot solids were strongly modified. In multilayered nanocomposite structure quantum dot decay lifetime was substantially shortened; the QD emission wa s enhanced by a factor of 4.4 , becoming highly anisotropic ; and the quantum e fficiency was increased to $30 \%$ in film. This strong plasmon coupling of excitons in such artificial three-dimensional plasmon resonator composites holds great promise for the achievement of highly efficient and anisotropic emitters for their possible end uses in the future optoelectronic and biophotonic technologies.

Acknowledgements: This work is supported by NRF -RF 2009-09, EU-FP7 Nanophotonics4Energy NoE, BMBF TUR 09/001, and TUBITAK EEEAG 107E088, 109E002, 109E004, and 110E010. HVD acknowledges support from ESF-EURYI and TUBA-GEBIP.

\section{References}

[1] Anker, J. N.; Hall, W. P.; Lyandres, O.; Shah, N. C.; Zhao, J.; Van Duyne, R. P. Nat. Mater. 2008, 7, 442-453.

[2] Gaponenko, S. V. Introduction to Nanophotonics; Cambridge University Press: Cambridge, UK, 2010.

[3] Komarala, V. K. et. al, A. Appl. Phys. Lett. 2006, 89, 253118.

[4] Sondergaard, T.; Jung, J.; Bozhevolnyi, S. I.; Della Valle, G. New J. Phys. 2008, 10, 105008.

[5] Muskens, O. L.; Giannini, V.; Sanchez-Gil, J. A.; Gomez Rivas, J. Opt. Express 2007, 15, 17736-17746. 